\title{
Juvenile neurogenesis makes essential contributions to adult brain structure and plays a sex-dependent role in fear memories
}

\author{
Jesse D. Cushman ${ }^{* * \neq}$, Jose Maldonado ${ }^{2+\neq}$, Eunice E. Kwon ${ }^{2+}$, A. Denise Garcia $^{2+}{ }^{\text {, Guoping Fan }}{ }^{3+}$, \\ Tetsuya Imura ${ }^{2 t}$, Michael V. Sofroniew ${ }^{2 t \neq}$ and Michael S. Fanselow ${ }^{1+\neq}$
}

${ }^{1}$ Departments of Psychology and Psychiatry and Biobehavioral Sciences, University of California Los Angeles, Los Angeles, CA, USA

2 Department of Neurobiology, University of California Los Angeles, Los Angeles, CA, USA

${ }^{3}$ Department of Human Genetics, University of California Los Angeles, Los Angeles, CA, USA

\section{Edited by:}

Susan J. Sara, Collège du France,

France

Reviewed by:

Marie H. Monfils, University of

Texas at Austin, USA

Stefano Sensi, University of

California Irvine, USA

*Correspondence:

Jesse D. Cushman, Departments of Psychology and Psychiatry and

Biobehavioral Sciences, University

of California Los Angeles,

8548 Franz Hall, Los Angeles,

CA 90095-1563, USA.

e-mail:jcushman@ucla.edu;

sofroniew@mednet.ucla.edu;

fanselow@ucla.edu

${ }^{\dagger}$ Author Contributions:

Jesse D. Cushman, Jose

Maldonado, Michael V. Sofroniew,

Michael S. Fanselow designed

research. Jesse D. Cushman, Jose

Maldonado, Eunice E. Kwon, A.

Denise Garcia, Tetsuya Imura

conducted research. Guoping Fan

contributed materials. Jesse D.

Cushman, Jose Maldonado, Eunice

E. Kwon, A. Denise Garcia, Tetsuya

Imura, Michael V. Sofroniew,

Michael S. Fanselow analyzed data.

Jesse D. Cushman, Jose

Maldonado, Michael V. Sofroniew,

Michael S. Fanselow wrote the

paper.

${ }^{\ddagger}$ These authors contributed equally.
Postnatal neurogenesis (PNN) contributes neurons to olfactory bulb (OB) and dentate gyrus (DG) throughout juvenile development, but the quantitative amount, temporal dynamics and functional roles of this contribution have not been defined. By using transgenic mouse models for cell lineage tracing and conditional cell ablation, we found that juvenile neurogenesis gradually increased the total number of granule neurons by approximately $40 \%$ in OB, and by $25 \%$ in DG, between 2 weeks and 2 months of age, and that total numbers remained stable thereafter. These findings indicate that the overwhelming majority of net postnatal neuronal addition in these regions occurs during the juvenile period and that adult neurogenesis contributes primarily to replacement of granule cells in both regions. Behavioral analysis in our conditional cell ablation mouse model showed that complete loss of PNN throughout both the juvenile and young adult period produced a specific set of sex-dependent cognitive changes. We observed normal hippocampus-independent delay fear conditioning, but excessive generalization of fear to a novel auditory stimulus, which is consistent with a role for PNN in psychopathology. Standard contextual fear conditioning was intact, however, pre-exposure dependent contextual fear was impaired suggesting a specific role for PNN in incidental contextual learning. Contextual discrimination between two highly similar contexts was enhanced; suggesting either enhanced contextual pattern separation or impaired temporal integration. We also observed a reduced reliance on olfactory cues, consistent with a role for OB PNN in the efficient processing of olfactory information. Thus, juvenile neurogenesis adds substantively to the total numbers of granule neurons in $\mathrm{OB}$ and $\mathrm{DG}$ during periods of critical juvenile behavioral development, including weaning, early social interactions and sexual maturation, and plays a sex-dependent role in fear memories.

Keywords: adult neurogenesis, juvenile neurogenesis, postnatal neurogenesis, hippocampus, olfactory bulb, learning and memory, sex difference, fear conditioning

\section{INTRODUCTION}

New neurons are continuously generated in the juvenile and adult brain in two major regions, the subgranular zone (SGZ), which contributes neurons to the dentate gyrus (DG) of the hippocampus, and the sub-ventricular zone (SVZ), which contributes neurons to the olfactory bulb (OB). These new neurons integrate with embryonically generated neurons and make important, though incompletely understood, functional contributions to cognition and behavior (Deng et al., 2010; Lazarini and Lledo, 2011). Although neurogenesis in the adult brain has been under intense investigation for some time, neurogenesis during the juvenile period has received far less attention. Now classical studies reported that juvenile neurogenesis adds to the final adult complement of granule neurons in OB and DG (Altman and Das, 1965; Bayer et al., 1982), but neither the total numbers of granule neurons added, nor the temporal dynamics of that addition during juvenile development, have been defined. Furthermore, the term "adult" neurogenesis has been applied across studies with substantial differences in the age of the animal. A precise definition of the distinction between juvenile and adult neurogenesis is, therefore, needed in order to determine their potentially different structural and functional roles (e.g., Wei et al., 2011). The more inclusive term of postnatal neurogenesis (PNN) is a far more accurate description of field of adult neurogenesis as a whole and will be the primary term used here. 
In this study, we evaluated the structural and behavioral roles of PNN using quantitative morphometry and behavioral evaluations in combination with a transgenic loss of function model. For morphometric analyses we used quantitative, unbiased stereological procedures in combination with two well-characterized, transgenically targeted mouse models for either lineage analysis or near complete and permanent ablation of PNN while leaving embryonic neurogenesis intact, in order to define the temporal dynamics and precise contribution of PNN to the adult compliment of $\mathrm{OB}$ and $\mathrm{DG}$ granule neurons during the juvenile period.

Functionally, PNN has been implicated in odor-based learning in the OB (Lazarini and Lledo, 2011), and in the DG it has been implicated in learning and memory processes, as well as neuropsychiatric and neurological disorders (Deng et al., 2010; Sahay et al., 2011b). For behavioral evaluations, we used this same transgenic ablation methodology, and conducted an extensive analysis of Pavlovian fear conditioning to determine the conditions under which PNN is important for context fear; as well as an analysis of stimulus generalization, contextual discrimination, and reliance on odor cues. Both males and females were evaluated because sex differences in both the OB and DG PNN have been widely reported (Parducz and Garcia-Segura, 1993; Maren et al., 1994; Galea, 2008; Sakamoto et al., 2011).

\section{METHODS}

\section{ANIMAL CARE AND BREEDING}

All mice were housed at University of California, Los Angeles (UCLA) on a $12 \mathrm{~h}$ light/12 h dark cycle in groups of 2-4 and had free access to food and water. For morphological evaluations, 72 mice were used and for behavioral experiments, 251 mice were used. mGFAP-Cre Reporter mice were generated by breeding mGFAP-Cre mice of 73.12 line (Garcia et al., 2004) with Z/EG reporter mice (Novak et al., 2000). DNMT1-cKO mice were generated by breeding female DNMT1-loxP/mGFAP-Crenegative mice with male DNMT1-loxP/mGFAP-Cre-positive (line 73.12) mice, resulting in approximately half of the offspring being DNMT1-lox/mGFAP Cre-negative (referred to as control) and half DNMT1-loxp/mGFAP-Cre-positive (referred to as DNMT1-cKO). Quantitative PCR was used to ensure that only one copy of Cre-recombinase was present in the male breeder. This breeding strategy was used to ensure that maternal care was provided by control dams in order to avoid any possible deficiencies in the quality of maternal care provided by DNMT1-cKO mothers. For all behavioral experiments mice were between 3 and 5 months of age and experiments were conducted during the light phase. All control and DNMT1$\mathrm{cKO}$ mice were from the same hybrid C57Bl6, BALB/C background. All experiments were conducted in accordance with guidelines set by the National Institutes of Health and as mandated by the University of California, Los Angeles Office for the Protection of Research Subjects and the Chancellor's Animal Research Committee.

\section{NEUROSPHERE ASSAY}

Neurosphere cultures were prepared from periventriclar tissue (Imura et al., 2003). After 12 days in vitro, sphere numbers were counted or spheres were differentiated by plating on coated glass coverslips in basal media in the absence of added growth factors.

\section{BrdU}

Bromodeoxyuridine (BrdU, Sigma), was administered as intraperitoneal injections of $200 \mathrm{mg} / \mathrm{kg}$ spaced every $12 \mathrm{~h}$ followed by perfusion after 14 days (Garcia et al., 2004). Numbers of new neurons in the $\mathrm{OB}$ and DG were calculated by using stereology to count the total number of BrdU labeled cells and multiplying this value by the percentage of BrdU cells that also express NeuN as assessed by double labeling.

\section{HISTOLOGICAL AND BIOCHEMICAL PROCEDURES}

For all histological evaluations, mice were anesthetized by barbiturate overdose, fixed by transcardial perfusion and processed for histological sectioning for stereological evaluations or for immunohistochemistry using standard procedures and commercially available antibodies and reagents (see Detailed Methods). For stereological analysis, serially collected sections through entire brain regions were used as required for unbiased sampling (Gundersen et al., 1988). Primary antibodies used for immunohistochemistry were: mouse anti-NeuN (1:1000, Chemicon), rabbit anti-green fluorescent protein (1:1000; Molecular Probes), rabbit anti-Caspase 3 (1:200; Cell Signaling Technologies) Mouse anti-NeuN (1:2500, Chemicon), Sheep anti-BrdU (1:5760, Maine Biotechnology Services), Goat anti-doublecortin (1:5000, Santa Cruz), Mouse anti-PSA-NCAM (1:2000, Chemicon), Rabbit antiGFAP (1:20,000; DAKO), Sheep anti-IAP (1:20,000; L. Hutnik, UCLA). Western blot analysis was performed using standard procedures and commercially available antibodies and reagents (see Detailed Methods). Antibodies used were: goat anti-GFAP (1:5000, Dako); rabbit anti-actin (1:500; Sigma).

\section{STEREOLOGY, MORPHOMETRY, AND STATISTICS}

Stereology was conducted by using a modified optical fractionator probe (Gundersen et al., 1988) and stereological image analysis software (Stereo Investigator, MBF Bioscience, USA) operating a computer-driven stage motorized in the $x, y$, and $z$ axes (Zeiss, USA, Ludl, USA). Areas to be counted were traced at low power and counting frames were selected in an unbiased, systematic and random fashion by the analysis software. Cell nucleus tops were counted using a $100 \times$ objective (1.4 NA). The Stereo Investigator optical fractionator probe was applied to estimate the total number of cells in the granule cell layer (GCL) of OB and DG and in the medial habenular nucleus in control and DNMT1-cKO mice at 14 days, 3,12 , and 24 months of age in $n=6$ animals of each genotype at each age point.

Double fluorescent labeled neurons were counted in three dimensions by scanning confocal laser microscopy. Stacks of $1.0 \mu \mathrm{m}$ thick optical slices (frame size of $100 \times 100 \mu \mathrm{m}$ ) were collected through the $z$-axis $(10-30 \mu \mathrm{m})$ of immunofluorescent treated frozen brain sections. Only cells that were double labeled and entirely in the three dimensions of the $z$-stack were counted. Quantification of newly generated neurons was accomplished by counting the number of NeuN immunofluorescent-labeled cells that also labeled for reporter protein (green fluorescent protein [GFP]) in GFAP-Cre Reporter mice. Statistical evaluations were performed using Prism ${ }^{\circledR}$ (GraphPad, San Diego, CA). 


\section{FEAR CONDITIONING}

For all fear conditioning tasks animals were brought to a holding room in their home cages and left undisturbed for a minimum of $30 \mathrm{~min}$. Animals were then transported to the experimental rooms in their home cage, placed by hand into one of four conditioning chambers $(30 \times 25 \times 25 \mathrm{~cm}$; Med-Associates Inc. $)$, each in their own sound attenuating cubicle. The chambers could be modified by using different shock grids, plastic inserts, and the presence or absence of the background fan. The chambers were cleaned with $70 \%$ ethanol before conditioning and between animals. A metal pan containing a thin film of Windex was placed underneath the grid floors to provide an olfactory component to the context (Simple Green was used in the olfactory-based conditioning task). Freezing was recorded using an automated near infrared (NIR) video tracking equipment and computer software (VideoFreeze, Med-Associates Inc.). Video was recorded at 30 frames per second and the software calculated the noise (standard deviation) for each pixel in a frame by comparing its gray scale value to previous and subsequent frames. This produced an "activity unit" score for each frame. Based on previous validation by a human observer, freezing was defined as sub-threshold activity [set at 19 activity units (AU)] for longer than $1 \mathrm{~s}$. Percent freezing was then calculated as the number of seconds the animal was scored as freezing divided by the total time.

\section{AUDITORY CONDITIONING TASK}

Mice were conditioned using delay auditory conditioning (Quinn et al., 2009; Jacobs et al., 2010) where auditory CS offset and shock onset are contiguous. For this experiment a total of 38 mice were used: (Control: $n=20,11$ male, 9 female; DNMT1-cKO: $n=18,8$ male, 10 female). The conditioning context consisted of an unmodified conditioning chamber with a shock grid consisting of 32 stainless steel rods, $0.4 \mathrm{~cm}$ in diameter, alternating in height spaced $1 \mathrm{~cm}$ apart, with the background fan on. On Day 1 , mice were placed in the conditioning chamber and after 3 min either a pure tone $(2800 \mathrm{~Hz}, 85 \mathrm{~dB})$ or white noise $(80 \mathrm{~dB})$ was presented for $20 \mathrm{~s}$, immediately followed by a $2 \mathrm{~s} 0.65 \mathrm{~mA}$ foot-shock. This was then repeated four more times (i.e., Three minutes followed by an auditory stimulus-shock pairing) for a total of five shock presentations. Mice were left in the chamber for $3 \mathrm{~min}$ following the last shock. A common confound when testing auditory fear is the freezing levels are too high prior to presentation of the auditory stimulus and may even differ between experimental groups. In order to avoid this confound we followed a procedure previously developed by our lab (Jacobs et al., 2010) whereby fear to the conditioning chamber is first extinguished using 3 days of 15 min non-reinforced exposures (Days 2-4) and a single $15 \mathrm{~min}$ pre-exposure to a distinct, novel context (Day 5, this chamber had an A-frame insert, a white plastic panel covering the shock grids and the background fan was off). On Days 6 and 7 the auditory stimuli were presented in this context, in counterbalance order. These testing days followed identical parameters to the training day, except that shock was not presented. Half of the animals received the trained auditory CS on the first day (whether that was the pure tone or white noise) and half received the novel auditory CS on the first testing day (Day 6). The opposite auditory CS was then presented on the second testing day (Day 7). Freezing was quantified during the first $8 \mathrm{~min}$ on Day 2 for the context test. For the analysis of context fear extinction across Days 2-4 the entire $15 \mathrm{~min}$ period was analyzed on each day. For the auditory tests on Days 6 and 7, the 3 min prior to the first tone presentation (referred to as baseline fear) and the mean freezing during the five auditory CS presentations was analyzed.

\section{STANDARD AND PRE-EXPOSURE-DEPENDENT CONTEXT FEAR TASK}

The conditioning chamber for these experiments was modified with two white plastic inserts that created a continuous curve of side and back walls. For the standard context fear task a total of 38 mice were used (Control: $n=19,9$ male, 10 female; DNMT1cKO: $n=19 ; 9$ male, 10 female). For the pre-exposure-dependent task a total of 99 mice were used (for pre-exposed groups; Control: $n=26,13$ male and 13 female; DNMT1-cKO: $n=19$, 9 male, 10 female; for non-pre-exposed groups: Control $n=27$, 15 male, 12 female; DNMT1-cKO: $n=17,8$ male, 9 female. The shock grid consisted of 16 stainless steel rods of alternating diameter $(0.4$ and $1.0 \mathrm{~cm}$ ) spaced $1.5 \mathrm{~cm}$ apart (center to center) and the background fan was on. Standard context conditioning: Day 1: mice received a $2 \mathrm{~s}, 0.75 \mathrm{~mA}$ foot-shock $5 \mathrm{~min}$ after placement in the chamber and were removed $30 \mathrm{~s}$ after the shock, Day 2: mice were returned to the identical conditioning chamber for the 8 min context test. Pre-exposure-dependent context conditioning: Day 1: mice were placed in the chamber for $5 \mathrm{~min}$, Day 2: mice were returned to the identical chamber for $10 \mathrm{~s}$ before foot-shock $(2 \mathrm{~s}, 0.75 \mathrm{~mA})$. They remained in the chamber for $30 \mathrm{~s}$ (42 s total) and were then returned to their home cages, Day 3: mice were returned to the identical conditioning chamber for the 8 min context test.

\section{CONTEXT FEAR DISCRIMINATION TASK}

This task involved training mice to fear one context (referred to as the $\mathrm{S}+$ context) but not another similar context (referred to as the $\mathrm{S}-$ context). For this experiment a total of 42 mice were used (Control: $n=20$ mice, 8 male, 12 female; DNMT1-cKO: $n=22$, 11 male, 11 female). The contexts were made very similar based on previous data indicating the difficult discriminations are more sensitive to impaired synaptic plasticity within the DG (McHugh et al., 2007). The exact same conditioning chamber and shock grids ( 32 stainless steel rods, $0.4 \mathrm{~cm}$ in diameter, alternating in height spaced $1 \mathrm{~cm}$ apart) were used for both $\mathrm{S}+$ and $\mathrm{S}-$. Only two features differed: the S+ context had a black plastic Aframe insert (consisting of two black plastic pieces attached by a hinge that sloped up from the side walls at $60^{\circ}$ ) and the background fan was on. On Days 1-3 of training, mice were placed into the conditioning context for a $4 \mathrm{~min}, 2 \mathrm{~s}$ session. Three minutes into the session, a $2 \mathrm{~s}, 0.5 \mathrm{~mA}$ shock was delivered. The mice were left in the context for another minute after the shock. The dependent measure was freezing behavior within the first 3 min (i.e., pre-shock) of each session. Freezing was measured during the $3 \mathrm{~min}$ prior to shock on each day. Generalization test: Days 4 and 5 of testing involved two 3 min sessions separated by 1.5-2 h. Mice were exposed to both $\mathrm{S}+$ and $\mathrm{S}-$ contexts in a counterbalanced order, with no shock delivered. On Day 4 the order of 
placement was counterbalanced so that half were placed in $\mathrm{S}+$ first and half in S- first. On Day 5 the order of placement was reversed for each mouse. Contextual fear discrimination training: Days 6-17 of testing consisted of discrimination training where animals were placed in both the $\mathrm{S}+$ and $\mathrm{S}-$ contexts each day. Again, in the $\mathrm{S}+$ context, mice received a $2 \mathrm{~s}, 0.5 \mathrm{~mA}$ shock after $3 \mathrm{~min}$ and were left in the chamber for $1 \mathrm{~min}$ following shock. In the $\mathrm{S}-$ context, mice were simply placed in the chamber for an equivalent $4 \mathrm{~min}$ and $2 \mathrm{~s}$. Freezing was measured during the 3 min preceding shock on all days in which shock was administered and the equivalent period of time in the $\mathrm{S}-$ context. The order of training followed a double alternation schedule: Day 6 $\mathrm{S}-$ then $\mathrm{S}+$, Day $7 \mathrm{~S}+$ then $\mathrm{S}-$, Day $8 \mathrm{~S}+$ then $\mathrm{S}-$, Day 9 $\mathrm{S}-$ then $\mathrm{S}+$, etc. For statistical analysis and graphical presentation the data was collapsed into consecutive 2 day blocks so that each block consisted of 1 day of $\mathrm{S}+$ then $\mathrm{S}-$ and 1 day of $\mathrm{S}-$ then $\mathrm{S}+$.

\section{ODOR-BASED DISCRIMINATION TASK}

For this experiment a total of 42 mice were used (Control: $n=23$, 13 male, 10 female; DNMT1-cKO: $n=19,9$ male, 10 female). On Days $1-5$ of training, mice were placed into the conditioning context for a $4 \mathrm{~min}, 2 \mathrm{~s}$ session. The conditioning context for this task was the same unmodified chamber used in the auditory fear task. The conditioning context consisted of an unmodified conditioning chamber with a shock grid consisting of 32 stainless steel rods, $0.4 \mathrm{~cm}$ in diameter, alternating in height spaced $1 \mathrm{~cm}$ apart, with the background fan on and Windex placed in the bottom pan as background odor). Three minutes into the session, a $2 \mathrm{~s}$, $0.65 \mathrm{~mA}$ shock was delivered. The mice were left in the context for another minute after the shock. The dependent measure was freezing behavior within the first 3 min (i.e., pre-shock) of each session. Freezing was measured during the $3 \mathrm{~min}$ prior to shock on each day. For the generalization test on Days 6 and 7 mice were exposed to the training context and a novel context for $3 \mathrm{~min}$, separated by $1.5-2 \mathrm{~h}$, in a counterbalanced order. The novel context was modified with an A-frame insert, the background fan was off and a different odor, Simple Green Cleaner, was used as the background odor. On Day 8 mice were again placed in both contexts, in a counterbalanced order, however, the background odors were switched for each context, i.e., Simple green was placed in the training context and Windex was placed in the novel context.

\section{MODIFIED SHIRPA}

A separate cohort of mice (Control: $n=18,9$ male, 9 female, DNMT1-cKO: $n=18$, 6 female, 12 male) was assessed using a modified version of the SHIRPA test battery (Rogers et al., 1997). The measures were: body length, body position, spontaneous activity, respiratory rate, tremor, urination, defecation, transfer arousal, piloerection, startle, gait, pelvic elevation, tail elevation, touch escape, positional passivity, trunk curl, limb grasp, abnormal behavior, grip strength, body tone, visual placing, pinna reflex, corneal reflex, wire maneuver, skin color, heart rate, limb tone, abdominal tone, salivation, provoked biting, righting reflex, contact righting reflex, negative geotaxis, fearfulness, irritability, aggression, and vocalization.

\section{RESULTS \\ PNN CONTINUALLY ADDS GRANULE NEURONS TO OB AND DG THROUGHOUT JUVENILE DEVELOPMENT}

To determine the total number of granule neurons added to $\mathrm{OB}$ and DG by PNN over time throughout the juvenile period we conducted two different types of quantitative morphometric analyses. For the first, we quantified the proportion of neurons generated by PNN at successive postnatal ages by using a transgenic mouse model for cell lineage mapping that selectively and specifically targets reporter protein labeling to essentially all neurons generated by PNN (Garcia et al., 2004). For the second, we used unbiased stereology to determine the total number of granule neurons in $\mathrm{OB}$ and DG at successive postnatal ages in wild-type mice.

To identify the proportion of neurons generated by PNN we crossed mice of mGFAP-Cre line 73.12 (Garcia et al., 2004) with Z/EG reporter mice (Novak et al., 2000). We have previously demonstrated that in the resulting mGFAP-Cre-Z/EG mice, $>95 \%$ of all granule neurons generated by PNN in adult $\mathrm{OB}$ and DG derive from reporter positive progenitors and can be identified by their expression of the lineage reporter molecule, GFP (Garcia et al., 2004). Using mGFAP-Cre-Z/EG mice, we quantified the ratio of neurons double-stained for GFP plus the neuronal marker, NeuN, relative to the total number of NeuN-stained neurons at various times between 7 days and 12 months of life as a means of estimating the relative contribution of neurons generated by PNN to the overall population of granule neurons in $\mathrm{OB}$ and DG. At postnatal day 7 (P7), there were no reporter positive neurons in either OB or DG. Thereafter, in the OB, postnatally generated granule neurons represented only $4.2 \%$ of all neurons at P14, increased in gradual increments to about $41 \%$ by 2 months and did not change significantly between 2, 3, and 12 months (Figure 1A). In the DG, postnatally born granule neurons represented only $3.2 \%$ of all neurons at P14, gradually increased to $22 \%$ by 2 months and did not change significantly between 2, 3, and 12 months (Figure 1B). Regression analysis of values between 7 days and 2 months indicated that slopes of increases in ratios of PNN-generated neurons were significant $(p<0.001)$ and incrementally linear over that time in both OB $\left(r^{2}=0.9712\right)$ and DG $\left(r^{2}=0.7886\right)$.

We next used unbiased stereology to determine the net contribution of PNN to the total number of granule neurons in adult $\mathrm{OB}$ and DG in control mice. We used cresyl violet staining, which allowed both the reliable collection of evenly spaced serial sections that are essential for rigorous stereological evaluation (Gundersen et al., 1988) and the reliable identification of granule neurons in both $\mathrm{OB}$ and DG as demonstrated by comparison of cell counts of neighboring sections stained with either cresyl violet or the neuronal marker NeuN (Figure 1C). Based on our observation that the number of mature reporter positive neurons derived from $\mathrm{PNN}$ in either $\mathrm{OB}$ or DG was negligible and only beginning at 2 weeks of age, we chose this time point as our first point of analysis to quantify the total numbers of mature neurons that derived from neurogenesis from embryonic progenitors. Optical Fractionator estimates showed that the total number of granule neurons increased significantly between 2 weeks and 2 months of age by $40 \%$ in $\mathrm{OB}$ and $24 \%$ in DG, and did not change 




B. Dentate gyrus ratio of PNN-generated granule neurons

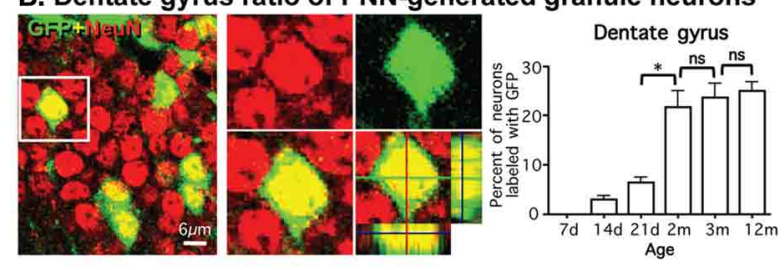

C. Stereology of granule neurons

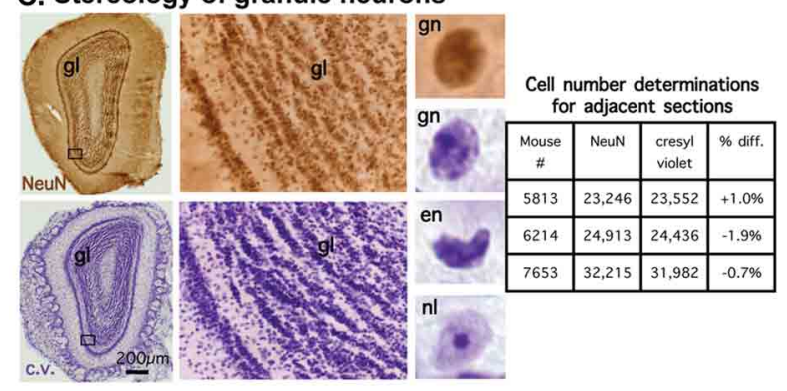

D. Postnatal increases in total numbers of granule neurons
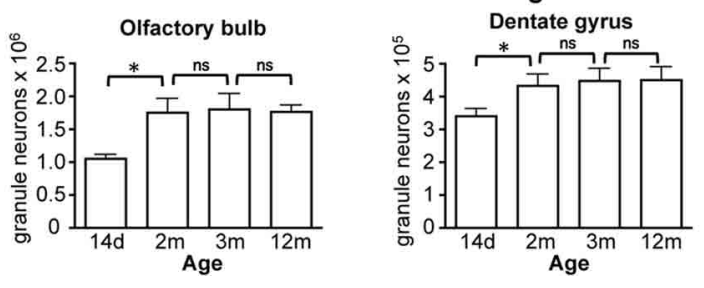

FIGURE 1 | Pronounced continual addition of granule neurons to $O B$ and DG throughout juvenile development. $(A, B)$ Gradually increasing ratio of postnatally generated neurons during postnatal development in $\mathrm{OB}$ and DG. Photomicrographs show confocal optical slices through OB (A) and DG (B) granule cell layers (GCL) from adult mGFAP-Cre-Z/EG reporter mice, stained green for the reporter, GFP, and red for the neuronal maker, NeuN. Graphs show ratios of granule neurons double-labeled for NeuN plus GFP, and therefore, derived from PNN, relative to the total population of NeuN labeled granule neurons at various postnatal ages in mGFAP-Cre-Z/EG reporter mice, expressed as \% (GFP + NeuN/NeuN $x$ 100). ${ }^{*} p<0.05$ ANOVA with Neuman-Keuls pairwise analysis; ns $=$ not significant; $n=3$ per group; $d=$ day; $m=$ month (C) Stereological analysis of cresyl violet (c.v.) and NeuN brightfield immunohistochemically stained $\mathrm{OB}$ tissue yield indistinguishable estimates of total numbers of granule neurons. Photomicrographs show surveys and details of adjacent $\mathrm{OB}$ frontal sections stained with immunohistochemistry for NeuN or c.v.; while c.v. labels moref cells, at high magnification granule neurons (gn) stained with NeuN or c.v. are morphologically the same and are easily distinguished from other cells, such as endothelia (en), cells with prominent nucleoli (nl) or small glia, which were not counted. Table shows that unbiased stereological cell counts of granule neurons stained with NeuN and c.v. are indistinguishable and do not differ significantly (ANOVA $p>0.05$ ).

(D) Significant increase in total numbers of granule neurons during postnatal development in OB and DG. Graphs show total numbers of granule neurons obtained using unbiased stereology in at various postnatal ages in OB and DG. ${ }^{*} p<0.05$ ANOVA with Neuman-Keuls pairwise analysis; $\mathrm{ns}=$ not significant; $n=4$ or 6 per group. significantly in either $\mathrm{OB}$ or DG between 2, 3, and 12 months (Figure 1D).

\section{A TRANSGENIC MOUSE MODEL FOR NEAR COMPLETE ABLATION OF PNN}

We next sought to generate a mouse model for near complete, permanent, and consistently reproducible ablation of PNN. To do so we looked for a means of transgenically targeting the conditionally mediated death of actively proliferating cells while sparing post-mitotic cells, using the cre-loxP system. The only known role of the DNA methyltransferase, DNMT1 is to methylate newly synthesized DNA (Jaenisch, 1997), which is critical for maintaining correct patterns of gene expression across multiple cell divisions. Loss of DNMT1 leads to apoptosis after 2-4 cell divisions (Jackson-Grusby et al., 2001) and its loss during embryonic development is lethal (Fan et al., 2001). However, restricting DNMT1 deletion to post-mitotic cells produces no observable effects (Fan et al., 2001). Because essentially all neural stem/progenitor cells that give rise to PNN begin to express GFAP in early postnatal period (Imura et al., 2003; Garcia et al., 2004), and because in the absence of injury many if not most astrocytes in vivo do not detectably express GFAP or do so after they are post-mitotic (Sofroniew, 2009), we reasoned that targeting the deletion of DNMT1 to GFAP + cells would allow the normal production of embryonically generated granule neurons in the $\mathrm{OB}$ and DG, but would cause a complete and permanent loss of PNN; and that non-proliferating post-mitotic GFAP-expressing astrocytes would be spared. We, therefore, crossed mice of mGFAP-Cre line 73.12 (Garcia et al., 2004) with mice in which exons 4 and 5 of the DNMT1 gene were flanked with loxP sites (Fan et al., 2001). We referred to the resulting mGFAP-Cre-DNMT1 mice as DNMT1-conditional knockout (cKO), or DNMT1-cKO, mice. Our line of reasoning predicts that the only expected effect in DNMT1-cKO mice should be the hypomethylation of DNA in highly proliferative progeny cells derived from the GFAP+ progenitors responsible for PNN. We tested this prediction in various ways. First, we identified cells in which DNMT1 function had been disrupted by conducting immunohistochemistry for intracisternal A particle (IAP), a marker for hypomethlyated DNA (Walsh et al., 1998; Fan et al., 2001). Consistent with our prediction, we found evidence of hypomethylation in the form of IAP immunoreactivity only in cells in the rostral migratory stream and the SGZ of the DG (Figure 9).

To determine the effect of DNMT1-cKO on PNN we evaluated formation of neurospheres in vitro from adult periventricular tissue (Imura et al., 2003) as well as by examining markers for immature neurons and incorporation of BrdU into granule neurons in adult mice in vivo (Garcia et al., 2004). Periventricular tissue from adult control mice yielded robust formation of pluripotent neurospheres $(43+5$ per $\mathrm{ml})$, whereas equivalent tissue from DNMT1-cKO mice yielded significantly fewer or no neurospheres $(2+1$ per $\mathrm{ml}, p>0.05, t$-test $)$ using well characterized procedures (Imura et al., 2003). In vivo, the markers for immature neurons, PSA-NCAM and doublecortin (DCX), were all almost entirely depleted in the $\mathrm{DG}, \mathrm{OB}$, and rostral migratory stream in adult DNMT1-cKO mice (Figure 10). In addition, BrdU incorporation into newly born granule neurons was reduced by more 
than $85 \%$ and $90 \%$ in OB and DG, respectively, in adult DNMT1cKO mice (Figure 2). Taken together, our findings indicate a near complete and permanent loss of PNN in both the SVZ and SGZ in DNMT1-cKO mice.

\section{NORMAL GROWTH AND APPEARANCE OF DNMT1-cKO MICE}

DNMT1-cKO mice did not differ significantly from controls in either body weight or brain weight at 3,12 , or 24 months $(n=6$ or more for each measure at each time point, $p>0.05$, ANOVA). There was no detectable difference in the cytoarchitecture of cresyl violet stained sections of forebrain regions that did not exhibit PNN in DNMT1-cKO mice as compared with controls (data not shown).

\section{NO DETECTABLE EFFECT OF DNMT1-cKO ON MATURE ASTROCYTES}

To evaluate astroglia we used immunohistochemical staining and western blotting for GFAP. Quantitative levels of GFAP determined by Western blotting as well as the morphological appearance of GFAP+ astroglia in all forebrain regions examined, including $\mathrm{OB}$, thalamus, and hippocampus were indistinguishable between DNMT1-cKO mice and controls (Figure 3). Together, our findings indicate that DNMT1-cKO mice exhibit a selective, near complete, and permanent ablation of PNN without detectable deleterious effects on other brain cells or cytoarchitecture.

\section{ABLATION OF PNN REDUCES ADULT GRANULE NEURONS BY 45\% IN OB AND BY $25 \%$ IN DG}

We next used unbiased stereology as described above to determine the net contribution of PNN to the total number of granule neurons in adult OB and DG in control and DNMT1-CKO mice. Optical Fractionator estimates showed that total neuronal numbers in both the OB and DG did not differ significantly between DNMT1-cKO and control mice at 14 days of age (Figures 4A-F). In control mice, the total number of granule neurons increased significantly between P14 and 3 months by $45 \%$ in the OB and $25 \%$ in the DG, and thereafter did not change significantly in either $\mathrm{OB}$ or DG at 12 or 24 months (Figures 4C,F). In contrast, in DNMT1-cKO mice, the total numbers of granule neurons were not significantly different at P14 days and 3 months in both OB and DG, and by 12 and 24 months the numbers had declined negligibly below the level at P14 (Figures 4C,F).

\section{NORMAL POSTNATAL DEVELOPMENT IN NON-PNN REGIONS OF DNMT1-CKO MICE}

To test whether DNMT1-cKO mice had normal numbers of neurons and normal postnatal structural development in forebrain regions that did not exhibit PNN, we conducted unbiased stereological evaluations of the medial habenular nucleus in control and DNMT1-cKO mice (Figures 4G-I). We chose the medial habenula because it is close to the DG, could be evaluated in exactly the same tissue sections used for DG evaluations, and because it has easily defined, unambiguous boundaries. Optical Fractionator estimates showed that total neuronal numbers in the medial habenula were indistinguishable between DNMT1-cKO and control mice at all time points examined, 2 weeks, 3, 12, and 24 months of age (Figure 4I). It is noteworthy that in both control and DNMT1-cKO mice, the medial habenula exhibited a small


FIGURE 2 | Depleted ability to generate new granule neurons in OB and DG of adult DNMT1-cKO mice. (A-D) Fourteen days after i.p. injection, BrdU labels many newly generated cells in granule cell layer (GCL) of $\mathrm{OB}$ in control $(\mathbf{A}, \mathbf{C})$ but few in DNMT1-cKO $(\mathbf{B}, \mathbf{D})$ adult mice. EPL, external plexiform layer (E) Confocal optical slice through an adult born neuron in GCL of OB stained green for the neuronal maker, NeuN and red for BrdU. (F) Stereological analysis showed a pronounced and statistically significant $85 \%$ reduction in the generation of new neurons in adult OB GCL of DNMT1-cKO mice relative to controls, $p<0.001$, $t$-test, both groups $n=6$. (G-J) BrdU labels many newly generated cells 14 days after

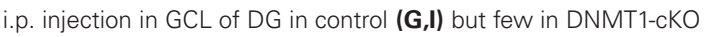
$\mathbf{( H , J )}$ adult mice. (K) As in E but for neuron in GCL of DG. (L) Stereological analysis showed a pronounced and significant $90 \%$ reduction in the generation of new neurons in adult DG GCL of DNMT1-CKO mice relative to controls, $p<0.001$, $t$-test, both groups $n=6$. 


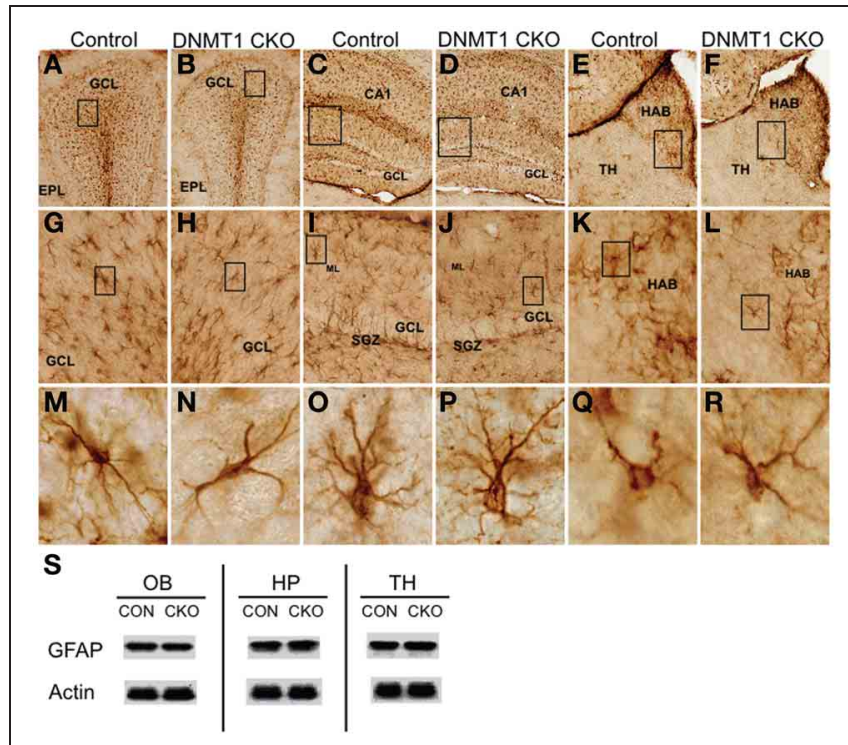

FIGURE 3 | Normal appearance of mature astrocytes and normal levels of GFAP in DNMT1-cKO mice. (A-R) Survey and detail of frontal sections through the olfactory bulb (A,B), hippocampus $(\mathbf{C}, \mathbf{D})$, and medial habenular nucleus (E,F) stained for GFAP. DNMT1-cKO tissue exhibits no detectable difference in the number or distribution GFAP positive astrocytes at the survey (A-F), intermediate (G-L), or single cell (M-R) levels in any area. Boxes indicate region shown at higher magnification in image below. Note that GFAP-positive progenitors in the subgranular zone (SGZ) of dentate gyrus (DG) are depleted as expected in DNMT1-cKO mice (J) as compared with control mice (I). (S) Western blots show that levels of GFAP are indistinguishable between controls and DNMT1-cKO mice in olfactory bulb $(\mathrm{OB})$, hippocampus $(\mathrm{HP})$, and thalamic $(\mathrm{TH})$ tissue. $\mathrm{GCL}$, granule cell layer; $\mathrm{EPL}$, external plexiform layer; $\mathrm{HAB}$, habenula; $\mathrm{ML}$, molecular layer; $\mathrm{SGZ}$, subgranular zone; Con, control; CKO, conditional knockout.

but significant decline in neuronal number of $16.5 \%$ between 2 weeks and 3 months.

\section{ABSENCE OF SEX DIFFERENCE IN GRANULE CELL NUMBERS IN CONTROL OR CKO MICE}

We also looked for potential sex differences in neuronal numbers or ablation efficiency. We found no difference in the total number of granule neurons between males and females for both the control and cKO mice (ANOVA for 3 and 12 month old mice, for OB: main effect of sex: $F(1,24)=0.008, p=0.930$; geno $\times$ sex interaction: $F(1,24)=0.921, p=0.351$; for DG: main effect of sex: $F(1,23)=0.002, p=0.969$; geno $\times$ sex interaction: $F(1,23)=$ $0.685, p=0.421$ ). This indicates that the efficiency of our ablation methodology was equivalent in males and females.

\section{BEHAVIORAL CHARACTERIZATION OF DNMT1-CKO MICE}

In order to assess the significance of juvenile neurogenesis to adult brain function we assessed behavior in young adult (3-5 months old) DNMT1-cKO mice. Our morphological findings indicate that these mice have a near complete ablation of neurogenesis throughout the postnatal period. They lack both the major structural addition during the juvenile period, as well as the much smaller adult generated contribution. Consistent with the specificity of the morphological observations described above,
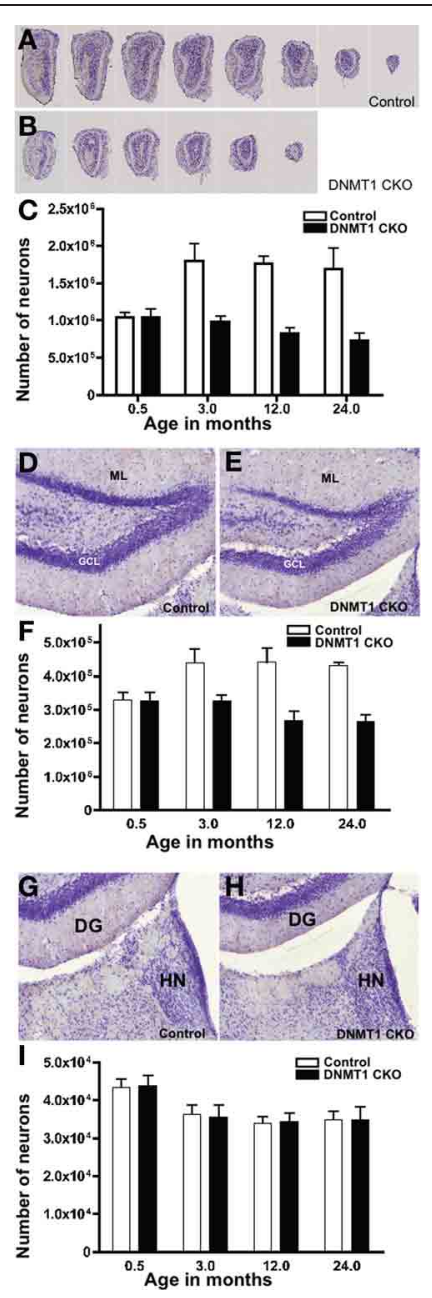

FIGURE 4 | Failure of postnatal addition of granule neurons to $O B$ and DG but normal development of medial habenula in DNMT1-cKO mice. $(\mathbf{A}, \mathbf{B})$ Evenly spaced sections through $\mathrm{OB}$ demonstrate the pronounced reduction in macroscopic size of OB in an adult DNMT1-cKO (B) mouse in comparison with an age-matched control (A). (C) Stereological analysis showed a pronounced and statistically significant $(p<0.001) 45 \%$ increase in the total number of GCL neurons in OB of control mice between 0.5 and 3 months of age, with no further significant change at 12 or 24 months. In the contrast, the total number of GCL neurons in OB of DNMT1-CKO mice did not change significantly between 0.5 and 3 months of age and declined slightly at 12 and 24 months (ANOVA with Neuman-Keuls pairwise analysis, all groups $n=6$ ). (D,E) The DG granule cell layer $(G C L)$ in an adult DNMT1-CKO (E) mouse is visibly thinner in comparison with an age-matched control (D). ML, molecular layer. (F) Stereological analysis showed a pronounced and statistically significant $(p<0.001) 25 \%$ increase in the total number of GCL neurons in DG of control mice between 0.5 and 3 months of age, with no further significant change at 12 or 24 months. In contrast, the total number of GCL neurons in DG of DNMT1-cKO mice did not change significantly between 0.5 and 3 months of age and declined slightly at 12 and 24 months (ANOVA with Neuman-Keuls pairwise analysis, all groups $n=6)$. $(\mathbf{G}, \mathbf{H})$ The medial habenular nucleus $(H N)$ in an adult DNMT1-cKO $(\mathbf{H})$ mouse does not differ visibly in appearance from that in an age-matched control (G). (I) Stereological analysis showed no statistically significant difference between the total numbers of neurons in $\mathrm{HN}$ of DNMT1-CKO and control mice at any age, but did reveal a significant $20 \%$ reduction between 0.5 months and all other ages ( $p<0.001$ ANOVA with Neuman-Keuls pairwise analysis, all groups $n=4)$ in both DNMT1-cKO and control mice. 
adult DNMT1-cKO mice did not exhibit any gross behavioral abnormalities in the SHIRPA phenotype assessment test or differences in shock sensitivity that would confound interpretation of behavioral experiments [as measured by activity units during shock presentation, averaged across the five shocks in the auditory fear conditioning task, control, and DNMT1-cKO mice did not differ $(F(1,45)=1.992, p=0.166)]$. For all of the following analyses, sex was included as a factor in the ANOVA and the results were broken down by sex when justified by a significant genotype $\times$ sex interaction. In order to provide sufficient power to resolve sex-dependent effects we ensured that there was a minimum of eight mice for each sex and genotype. As described in Figure 8 and analyzed below, we observed sex-dependent differences in reliance on olfactory cues. For the experiments that focused on hippocampus-dependent learning we, therefore, kept odor constant across any contextual manipulations.

\section{DNMT1-CKO MICE EXHIBIT NORMAL HIPPOCAMPUS-INDEPENDENT AUDITORY FEAR, BUT INCREASED GENERALIZATION TO A NOVEL AUDITORY STIMULUS}

In order to determine if hippocampus-independent learning and memory was affected in DNMT1-cKO mice we utilized a modified version of the standard auditory delay fear conditioning task, which allowed us to ensure that baseline levels of freezing prior to presentation of the auditory conditional stimulus (CS) were low and did not confound interpretation of the data (see Figure 5A for experimental design) (Jacobs et al., 2010). In addition, we were interested in testing the stimulus specificity of auditory fear by presenting both the auditory CS that was used during training as well as a novel auditory stimulus. Recent evidence has shown that although the hippocampus is not necessary for auditory delay fear conditioning it is involved in stimulus generalization, i.e., freezing to the trained stimulus is intact in animals with hippocampal lesions, however, generalization to a novel auditory CS is markedly reduced (Quinn et al., 2009). Half the animals were, therefore, conditioned with a pure tone $(2800 \mathrm{~Hz}$, $85 \mathrm{~dB})$ and half with white noise $(80 \mathrm{~dB}$; five $0.65 \mathrm{~mA}$ pairings). Over two testing days the "trained" and "novel" CS were presented in a counterbalanced order. As shown in Figure 5C baseline freezing prior to presentation of the auditory CS was low and did not differ between genotypes $(F(1,44)=2.39, p=$ $0.13)$. Freezing to the trained tone was normal in DNMT1cKO mice $(F(1,44)=0.688, p=0.412)$, however, freezing to the novel CS was significantly enhanced $(F(1,44)=5.149, p=$ 0.029). The ratio of freezing between the trained and novel CS, calculated as [trained/(trained + novel)] was also significantly reduced in the DNMT1-cKO mice $(F(1,44)=5.02, p=0.031$; Figure 5D). The similar level of freezing to the auditory CS indicates that hippocampus-independent fear conditioning is normal in DNMT1-cKO mice, consistent with previous neurogenesis ablation studies (Shors et al., 2002; Saxe et al., 2006; Drew et al., 2010). In addition, these results show that, opposite to the effect




of lesioning all subfields of the hippocampus (Quinn et al., 2009), loss of PNN results in increased stimulus generalization, e.g., an excessive level of freezing to a CS that was never paired with shock.

Inherent in the design of this procedure is both a test of contextual fear (Day 2) and a test of contextual fear extinction (Days 3-4). DNMT1-cKO mice showed no differences in contextual fear on Day 3 (Figure 4B, $F(1,44)=0.261, p=0.612$ ) and, although repeated measures ANOVA across Days 2-4 indicated significant extinction $(F(2,82)=8.624, p<0.001)$, this did not interact with genotype $(F(2,82)=1.732, p=0.183$, data not shown).

\section{DNMT1-CKO MICE EXHIBIT A SEX-SPECIFIC DEFICIT IN PRE-EXPOSURE-DEPENDENT CONTEXTUAL FEAR CONDITIONING}

The intact contextual fear in DNMT1-cKO mice was a surprising finding, given the $25 \%$ reduction in DG granule cell numbers in these mice. Recent work has demonstrated that contextual fear with multiple shocks is less sensitive to both hippocampal lesions (Wiltgen et al., 2006) and ablation of adult neurogenesis (Drew et al., 2010). We, therefore, sought to assess two forms of single-shock contextual fear, referred to here as "standard" and "pre-exposure dependent" contextual fear. In the standard contextual fear task, mice received a single $0.75 \mathrm{~mA}$ shock 5 min after placement in the conditioning chamber and were then brought back for an $8 \mathrm{~min}$ context test $24 \mathrm{~h}$ later (Figure 5A). As shown in Figure 6B, DNMT1-cKO mice performed normally in this task $(F(1,38)=0.002, p=0.961)$, consistent with our findings using multiple shocks.

In the pre-exposure dependent contextual fear task mice are pre-exposed to the context on Day 1, in the absence of shock, and then placed in the context the following day (Day 2) and administered a $0.75 \mathrm{~mA}$ shock $10 \mathrm{~s}$ after placement in the chamber (referred to as an "immediate" shock, see Figure 6C) (Fanselow,
1990). They are returned to the context on Day 3 for an 8 min context test. In this form of contextual fear the learning about the context occurs in the absence of shock and is, therefore, an example of "incidental" contextual learning, as no specific motivating stimuli or task demands are present (Tolman, 1948; Stote and Fanselow, 2004). An advantage of this task is that animals not pre-exposed to the context prior to the immediate shock exhibit the immediate shock deficit (Fanselow, 1990), that is they show very low levels of freezing because they have no contextual representation to associate the shock with (Fanselow, 1990; Rudy et al., 2004; Stote and Fanselow, 2004). The pre-exposed animals are rescued from this deficit because they are able to retrieve the representation formed during the pre-exposure and associate it with the immediate shock (Fanselow, 1990). The non-pre-exposed groups thus provide an ideal control condition that allows us to specifically isolate the contribution of contextual learning to the expression of freezing in the pre-exposed group.

As shown in Figure 6D, DNMT1-cKO mice exhibited a sexspecific impairment in pre-exposure dependent contextual fear, with pre-exposed male DNMT1-cKO mice exhibiting significantly less freezing than male controls (Genotype $\times$ Sex interaction: $F(1,44)=4.15, p=0.048$; Effect of genotype in males: $F(1,23)=5.829, p=0.025$; Effect of genotype in females: $F(1,22)=0.690, p=0.416)$. Furthermore, pre-exposure to the conditioning chamber failed to show any rescue of the immediate shock deficit in male DNMT1-cKO mice $(F(1,18)=0.276, p=$ $0.606)$, whereas for all other groups pre-exposure increased freezing relative to non-pre-exposed groups (male controls: $F(1,28)=$ 13.415, $p=0.001$; female controls: $F(1,25)=8.501, p=0.008$; female DNMT1-cKO: $F(1,21)=4.639, p=0.044)$. Freezing levels were equivalent in all non-pre-exposed groups, which rules out non-specific differences in the tendency to freeze (Effect of: Genotype: $F(1,44)=1.00, p=0.323$; Sex: $F(1,44)=0.190$,
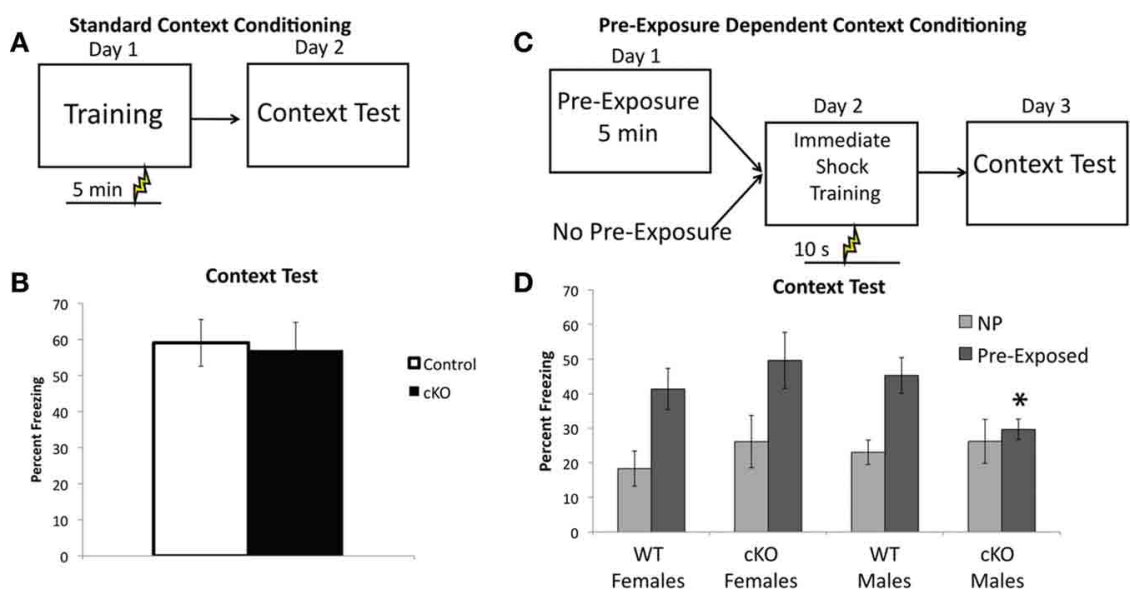

FIGURE 6 | DNMT1-cKO mice a sex-specific impairment of pre-exposure-dependent, but not standard contextual fear. (A) Experimental design for the Standard Contextual Learning. Shock is administered 5 min after placement in the conditioning chamber on the training day. Animals are returned to the context $24 \mathrm{~h}$ later for a context test. (B) Percent freezing in during the context test for the standard context fear task. (C) Experimental design for the Pre-exposure-dependent Context

Fear task. Mice were either pre-exposed to the conditioning chamber for 5 min or left in their home cage. Twenty-four hours later they received an immediate shock in the conditioning chamber and $24 \mathrm{~h}$ after that the mice received a context test. (D) Pre-exposed male DNMT1-cKO mice exhibited significantly less freezing than male controls (indicated by ${ }^{*}$ ) and failed to show the pre-exposure rescue of the immediate shock deficit exhibited by all other groups. 
$p=0.666$; Genotype $\times$ Sex: $F(1,44)=0.176, p=0.677)$. These findings indicate that loss of PNN is associated with a sex-specific deficit in pre-exposure-dependent, or "incidental" contextual fear, but normal performance in a standard, single-shock contextual fear task.

\section{DNMT1-CKO MICE EXHIBIT A SEX-SPECIFIC ENHANCEMENT IN A CONTEXTUAL FEAR DISCRIMINATION TASK}

The ability of mice to discriminate between two similar contexts has been previously shown to depend on intact NMDA receptor mediated synaptic plasticity specifically within the DG and is thought to be a behavioral test of pattern separation ( $\mathrm{McHugh}$ et al., 2007). In contextual fear discrimination mice are first trained to fear one context (referred to as S+, Figure 7A, $0.5 \mathrm{~mA}$ shock 3 min after placement in the context) and then go through a 2 day generalization test where freezing is assessed in both the $\mathrm{S}+$ context and a novel context, referred to as the $\mathrm{S}-$ context (Figure 7C). Finally, the mice receive 12 days of discrimination training during which the $\mathrm{S}+$ context is paired with shock but the $\mathrm{S}-$ context is not (Figure 7E). For this experiment $\mathrm{S}+$ and $\mathrm{S}-$
A

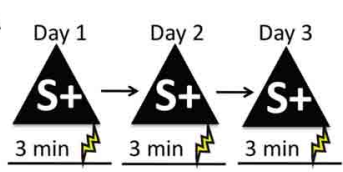

B

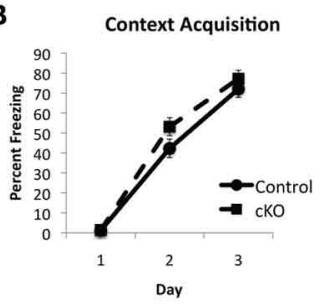

$\mathbf{J}$

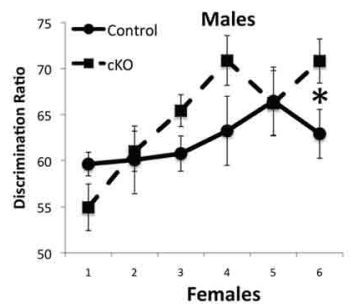

$\mathbf{L}$





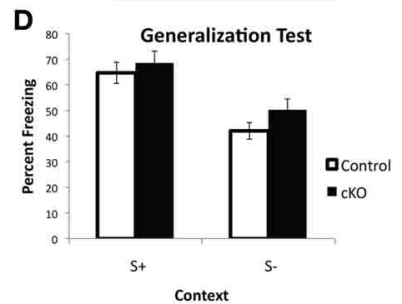

Context
$F$



H

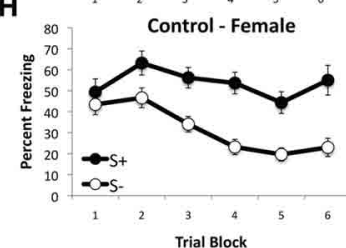

Change in Discrimination

$\mathbf{K}$
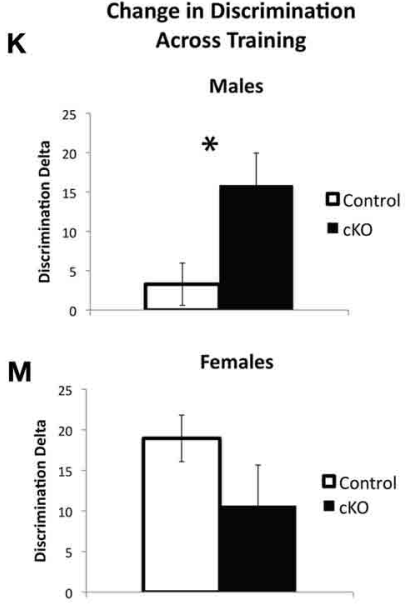

Trial Block 1 Trial Block 2 Trial Block 3 Trial Block 4 Trial Block 5 Trial Block 6

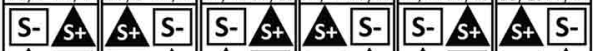

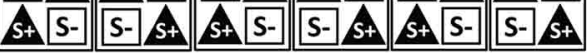

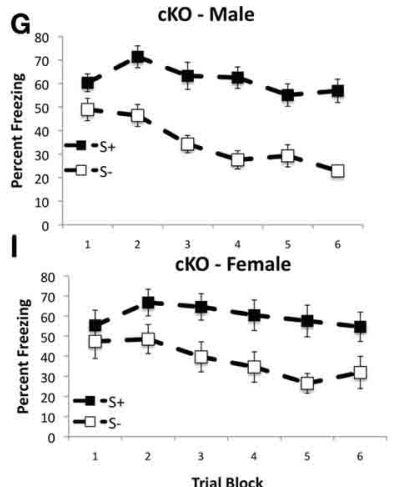

FIGURE 7 | DNMT1-cKO mice show a sex-specific enhancement in context discrimination learning. (A) Experimental design for the acquisition of fear to the $\mathrm{S}+$ context. Mice were placed in the conditioning chamber and shocked 3 min later. (B) DNMT1-cKO mice show normal acquisition of contextual fear. (C) Experimental design for the generalization test. Mice were placed in both the S+ and S- contexts over 2 days, with the order of exposure counterbalanced. (D) DNMT1-cKO mice show normal levels of freezing in the $\mathrm{S}+$ context as well as generalization to the $\mathrm{S}-$ context.

(E) Experimental design for context discrimination training. Mice were placed in the S+ context, where they received a foot-shock after 3 min, and the Scontext, where they never received a foot-shock, over the course of 12 days. Order of presentation followed a double alternation schedule and each 2 day period was averaged together to make six Trial Blocks. ( $\mathbf{F}$ and $\mathbf{G}$ ) Freezing in $\mathrm{S}+$ and $\mathrm{S}-$ across discrimination training in male control and
DNMT1-cKO mice, respectively. Male controls failed to show any improvement in discrimination across training, whereas DNMT1-cKO mice did. (H and $\mathbf{~ I ) ~ F r e e z i n g ~ i n ~} \mathbf{S}+$ and $\mathbf{S}$ - across discrimination training in female control and DNMT1-cKO mice. Discrimination improved across training in both genotypes. (J) Discrimination ratios, calculated as freezing in $\mathrm{S}+/(\mathrm{S}++\mathrm{S}-$ ), in male control and DNMT1-cKO mice. DNMT1-cKO mice exhibited a significantly higher discrimination ratio on the final Trial Block. (K) Change in the discrimination ratio across training, calculated as the (discrimination ratio on Trial Block 1-discrimination ratio on Trial Block 6). Male DNMT1-cKO mice showed a significant enhancement (as indicated by $\left.{ }^{*}\right)$. (L) Discrimination ratios across training in females. No significant differences were observed. (M) Change in discrimination across training in females. No significant differences were observed. 
were made highly similar and differed in only two respects: the $\mathrm{S}+$ context had a plastic A-frame insert and the background fan was on, whereas in the $\mathrm{S}-$ context the A-frame was removed and the fan was turned off. All other contextual features were identical, such as the shock grid, cleaning solution, and background odor. Freezing was measured during the $3 \mathrm{~min}$ prior to shock presentation in the $\mathrm{S}+$ context and the equivalent time in the $\mathrm{S}-$ context.

DNMT1-cKO mice exhibited normal acquisition of contextual fear, consistent with our previous findings (repeated measures ANOVA for Day 2 and 3, Effect of: Day: $F(1,38)=81.02, p<$ 0.001 ; Day $\times$ genotype: $F(1,38)=0.422, p=0.520$; Day $\times$ sex: $F(1,38)=0.481, p=0.492$; Day $\times$ Genotype $\times$ Sex: $F(1,38)=$ $0.915, p=0.345)$. The generalization test showed that both control and DNMT1-cKO mice were able to distinguish the S+ from the $\mathrm{S}$ - context prior to discrimination training and showed similar levels of freezing in each (repeated measures ANOVA for S+ and S-, Effect of: Context: $F(1,38)=90.18, p<0.001$; Context $\times$ Genotype: $F(1,38)=1.29, p=0.263$; Context $\times$ Genotype $\times$ Sex: $F(1,38)=0.350, p=0.558)$.

Over the course of discrimination training, however, a sex-specific enhancement emerged in the male DNMT1cKO mice (repeated measures ANOVA with Trial block and Context, Effect of: Context: $F(1,190)=238.91, p<0.001$; Trial block: $F(5,190)=26.817, p<0.001$; Context $\times$ Trial block: $F(5,190)=9.942, p<0.001$; Context $\times$ Trial block $\times$ Geno $\times$ sex: $F(5,190)=2.785, p=0.019)$. Male DNMT1-cKO mice showed a significant benefit of discrimination training (as indicated by a significant Trial block $\times$ Context interaction: $F(5,50)=5.185, p=0.001)$, whereas male control mice did not (non-significant Trial block $\times$ Context interaction: $F(5,35)=$ $0.102, p=0.991)$. The discrimination ratio [calculated as: $\mathrm{S}+/(\mathrm{S}++\mathrm{S}-)]$ was significantly higher in the male DNMT1$\mathrm{cKO}$ relative to control mice on the final Trial Block $(F(1,19)=$ $4.814, p=0.042$, Figure 7A) and the change in discrimination between the first and last Trial Blocks was also higher in the male DNMT1-cKO mice $(F(1,19)=5.235, p=0.035)$. In females, both the controls and DNMT1-cKO mice showed a significant increase in discrimination across training (Trial Block $\times$ Context interaction: $F(5,55)=9.326, p<0.001 ; F(5,50)=$ $3.761, p=0.006$, respectively). Their discrimination ratios were similar across training (Figure 7B) and the change in the discrimination ratio across training (Figure 7D) also did not differ $(F(1,23)=2.715, p=0.114)$. These findings indicate that loss of PNN is associated with a sex-specific enhancement of contextual discrimination learning. This enhancement is driven by the fact that male control mice do not benefit from 12 days of discrimination training on this difficult task, whereas male DNMT1-cKO mice do.

\section{DNMT-CKO MICE EXHIBIT A SEX-DEPENDENT REDUCTION IN RELIANCE ON OLFACTORY CUES}

Previous studies on the functional role of OB PNN have consistently reported no effect on odor detection, however, its involvement in learning about new odors and discrimination between odors is much more mixed (Lazarini and Lledo, 2011). We, therefore, sought to determine the extent to which loss of PNN affects reliance on olfactory cues in contextual fear conditioning. Mice were first trained to fear a context over 5 days with a single $0.65 \mathrm{~mA}$ shock $3 \mathrm{~min}$ after placement into the context on each day (Figure 8A). DNMT1-cKO mice again showed normal acquisition of contextual fear (Repeated measures ANOVA for pre-shock freezing on days 2-5: Overall effect of Day: $F(3,102)=$ 72.198, $p<0.001$ : Genotype $\times$ day interaction: $F(3,102)=$ $1.112, p<0.348$ ); (Figure 8B). We then conducted a generalization test where mice were placed in both the training context and a distinctly different novel context, with order counterbalanced, over 2 days (Figure 8C). The training and novel contexts were made very distinct from each other and were in different rooms, but were also characterized by different odors: the training context was scented with Windex ${ }^{\circledR}$ Original Cleaner whereas the novel context was scented with Simple Green ${ }^{\circledR}$. As can be seen in Figure 8D, both control and DNMT1-cKO mice showed similar levels of freezing to the trained context and equivalent generalization to the novel context (overall effect of context: $F(1,34)=116.59, p<0.001$; no interaction with genotype: $F(1,34)=0.896, p=0.351)$. On the final day, mice were again returned to both the training and novel contexts, but this time the odors were reversed (i.e., Simple Green in the training context and Windex in the novel context; Figure $\mathbf{8 E}$ ). The behavioral impact of this odor switch was used to analyze the extent to which control and DNMT1-cKO mice rely on odor in distinguishing the two contexts. The odor reversal significantly reduced freezing overall (RM ANOVA with generalization test and odor reversal test; overall effect of test $F(1,34)=33.58$, $p<0.001$ ) but this decrement strongly depended on context, genotype and sex (test $\times$ context $\times$ genotype $\times$ sex interaction: $F(1,34)=12.496, p=0.001)$. In order to more fully characterize this interaction we calculated a difference score for the extent to which freezing decreased in $\mathrm{S}+$ by use of the $\mathrm{S}-$ odor (Freezing in $\mathrm{S}+$ for the Generalization test - Freezing in $\mathrm{S}+$ for the Odor Reversal) and, conversely, the extent to which freezing was altered in the $\mathrm{S}-$ context by use of the $\mathrm{S}+$ odor (Freezing in $S$ - for the Generalization test - Freezing in $S+$ for the Odor reversal test). In the $\mathrm{S}+$ context, male DNMT1-cKO mice failed to show the odor reversal-induced freezing decrement that was exhibited by the male control mice $(F(1,17)=5.504, p=0.031)$, whereas female DNMT1-cKO mice were similar to controls $(F(1,17)=0.058, p=0.813)$. In the $S-$ context, the odor reversal reduced freezing in female controls, but not female DNMT1cKO mice (effect of genotype; $F(1,17)=7.137, p=0.016$ ), whereas male control and DNMT1-cKO mice were not affected by the odor reversal and did not differ from each other $(F(1,17)=$ $0.452, p=0.510)$. Overall, these findings indicate that loss PNN reduced the behavioral impact of manipulating odor on the expression of contextual fear, suggesting a reduced reliance on olfactory cues.

\section{DISCUSSION}

Taken together, our quantitative morphometric analyses show that during the period between 14 days and 2 months, juvenile neurogenesis makes a substantive contribution to the development of the OB and DG by adding approximately $40 \%$ and $25 \%$, respectively, of their total adult compliment of granule cells. This 
A

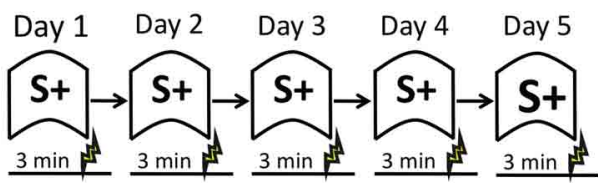

C

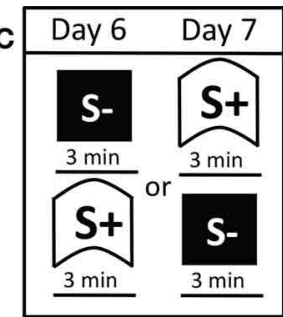

Generalization Test

B

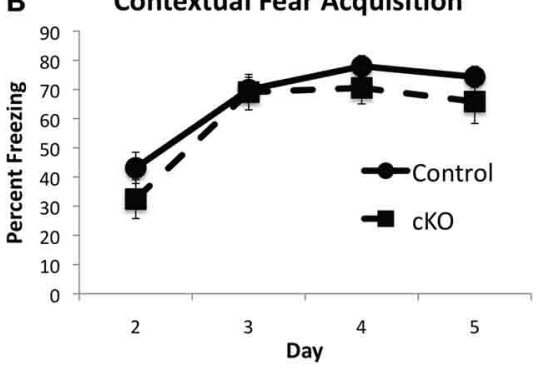

D

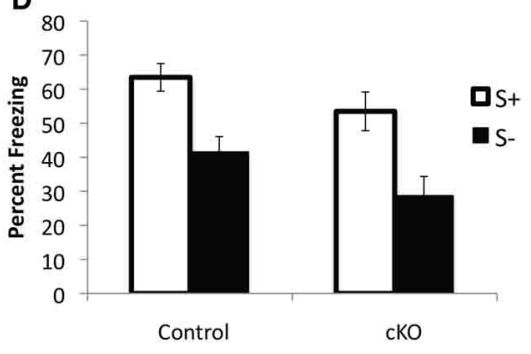

Odor Reversal in S+

E
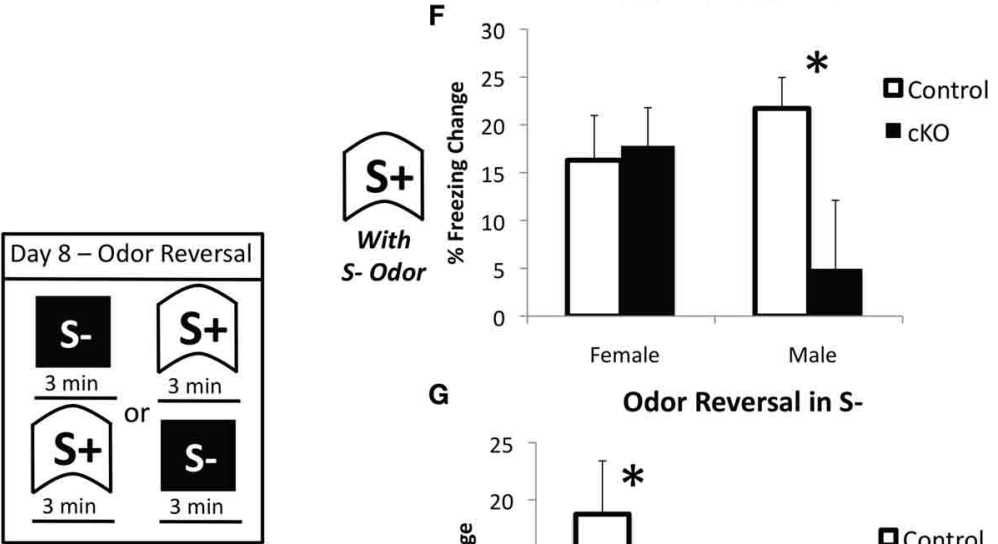

G

Odor Reversal in S-

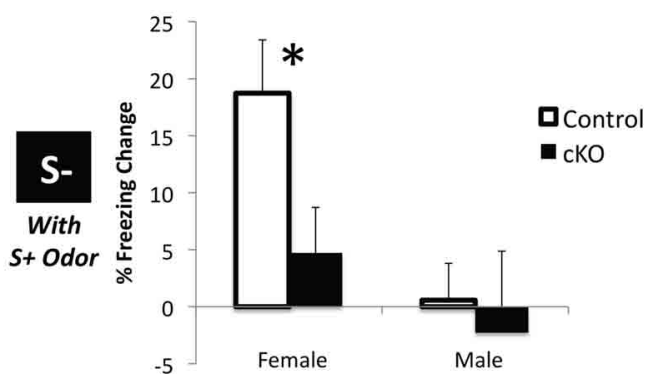

FIGURE 8 | DNMT1-cKO mice exhibit a sex-dependent impairment in odor-based discrimination. (A) Experimental design for the acquisition of fear to the training $(\mathrm{S}+$ ) context. Mice were placed in the conditioning chamber and shocked 3 min later. (B) DNMT1-cKO mice exhibited normal context fear acquisition. (C) Experimental design for the generalization test. Mice were placed in both the $\mathrm{S}+$ and $\mathrm{S}-$ contexts over 2 days, with the order of exposure counterbalanced. These two contexts were structurally distinct and characterized by Windex and Simple Green odor, respectively. (D) DNMT1-cKO mice showed normal levels of freezing to the $\mathrm{S}+$ context and equivalent generalization to the $\mathrm{S}$ - context. (E) Experimental design for the odor reversal test on Day 8. Mice were again placed in both
$\mathrm{S}+$ and $\mathrm{S}-$ in counterbalanced order, but the odors were reversed (i.e., Windex was used in $\mathrm{S}-$ and Simple Green in S+). (F) Effect of odor reversal in $\mathrm{S}_{+}$, the difference score was calculated as freezing in $\mathrm{S}+$ with odor intact-freezing in $\mathrm{S}$ - with odor reversed. The odor reversal reduced freezing in all but the male DNMT1-cKO mice, which showed a significant reduction relative to male control mice (as indicated by*).

(G) Effect of odor reversal in $\mathrm{S}-$, the difference score was calculated as freezing in $\mathrm{S}$ - with odor intact-freezing in $\mathrm{S}$ - with odor reversed. The odor reversal reduced freezing in female control mice only. Female DNMT1-cKO mice were significantly different relative to female controls (as indicated by *). addition is made on top of a relatively stable population of embryonically generated neurons that are not replaced in substantive numbers. Our findings further show that total granule cell numbers do not differ significantly at 2, 3, 12, and 24 months of age in both $\mathrm{OB}$ and DG, indicating that the total adult compliment of granule neurons remains stable in wild-type mice over their entire adult life span, including aging. Given that the number of embryonically generated neurons is stable and after 2 months the 


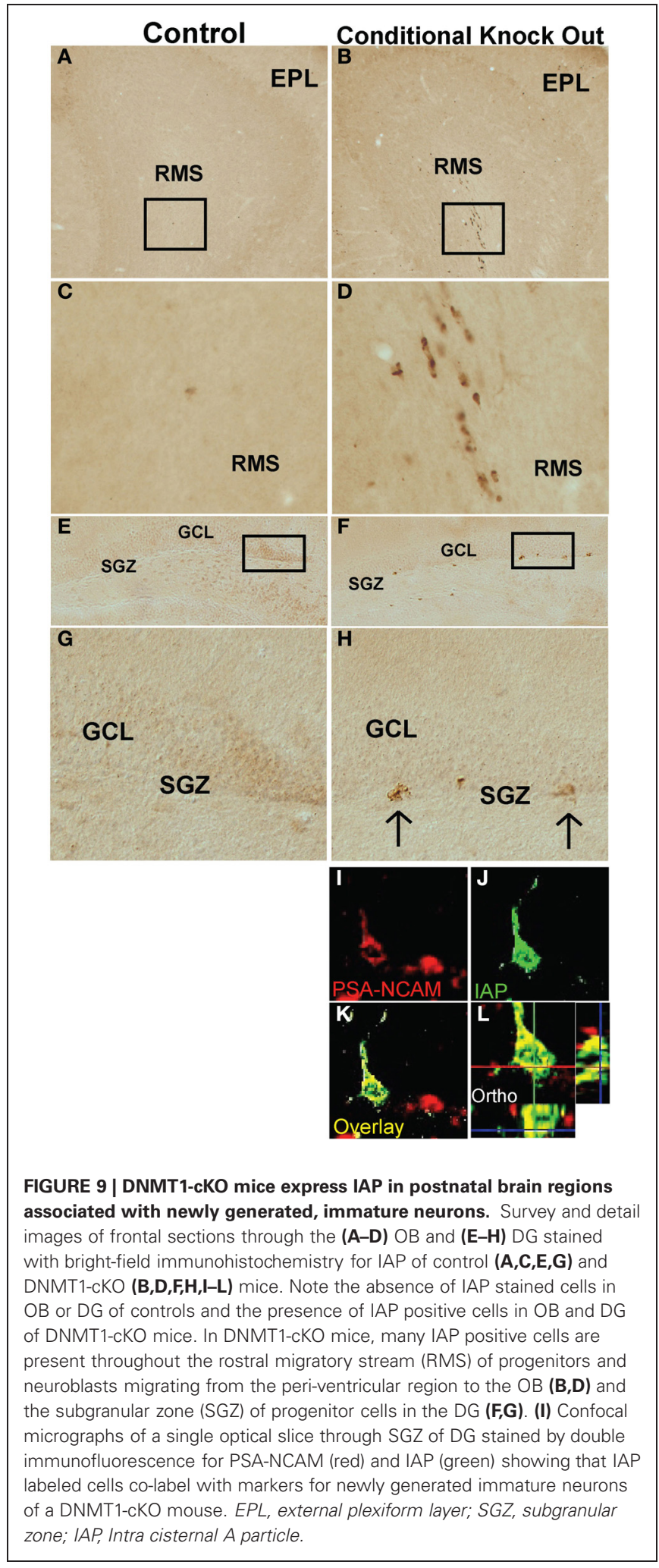

total number of DG and OB neurons does not increase, adult neurogenesis must preferentially replace juvenile or adult generated, rather than embryonically generated, neurons. In addition, we found no detectable sex differences in either the total numbers



FIGURE 10 | Expression of PSA-NCAM and Doublecortin is depleted in the $O B$, rostral migratory stream (RMS), DG, and subgranular zone (SGZ) of DNMT1-cKO mice. Survey and detail of frontal sections through the OB (A-H), RMS (I-P), and DG (Q-X) stained with bright-field immunohistochemistry for PSA-NCAM (left panel) and Doublecortin (right panel). PSA-NCAM and Doublecortin labeled cells are rare in DNMT1-cKO mice as compared to age matched controls. Scale bars: $D, I, T=10 \mathrm{~mm} ; H, P, X=1.25 \mathrm{~mm}$.

of granule neurons in mature adults or in the numbers added to the $\mathrm{OB}$ or DG by PNN during juvenile development. It deserves emphasis, that in contrast to the $\mathrm{OB}$ and DG, which added neurons during the juvenile period in wild-type mice, the medial habenula exhibited a significant decline in neuronal number of $16.5 \%$ between 14 days and 3 months in a manner consistent with the canonical developmental model of over-production of neuronal numbers followed by pruning through naturally occurring cell death (Cowan et al., 1984). Thus, the continual addition 
of granule neurons in the $\mathrm{OB}$ and DG differs markedly from the developmental program that occurs in most other regions of the CNS. Our findings demonstrate that both the OB and DG continually add substantive numbers of neurons throughout periods of important juvenile behavioral development that include weaning, early environmental and social interactions, and sexual maturation.

Our findings are consistent with and extend older studies that used volumetric estimates of cell number to provide evidence for the addition of granule neurons to both $\mathrm{OB}$ and $\mathrm{DG}$ during the juvenile period (Bayer et al., 1982). These studies, conducted prior to the advent of modern stereological quantification and transgenic technology, did not provide accurate information for either total neuronal numbers or precise time course of their addition. Surprisingly, in spite of the surge of interest in adult neurogenesis over the past 20 years, little attention has been paid to neurogenesis during the juvenile period or to accurately documenting the degree to which juvenile neurogenesis might contribute in a developmental manner to the total compliment of granule neurons in adult OB or DG. There have been numerous studies documenting the proportional addition of new granule neurons to the $\mathrm{OB}$ or DG by using labeling with cell division markers or activation of genetically encoded lineage markers (Cameron and Mckay, 2001; Petreanu and Alvarez-Buylla, 2002; Kempermann et al., 2003; Lagace et al., 2007; Ninkovic et al., 2007; Imayoshi et al., 2008). These studies all differ substantively from ours in that they are reported by the authors to examine only adult (and not juvenile) neurogenesis and they examine only the relative proportions of labeled neurons. None of these previous studies provides information about total numbers of granule neurons or how this total number is influenced by PNN during either juvenile or adult life. Nevertheless, when carefully examining the reported ages of animals used in previous investigations, our findings are compatible with, and markedly extend, previous studies. For example, our findings indicate that "adult" neurogenesis in mice, when strictly defined as that occurring 2 months of age and older, contributes less than $1 \%$ of the total population of granule cells in the DG. This is consistent with Lagace et al., 2007, but is far less than the $5-6 \%$ estimate in juvenile rats from Cameron and Mckay, 2001. Our findings differ from the conclusion of Imayoshi et al., 2008, who suggest that the structural contribution of PNN differs between the OB and DG, contributing to continually ongoing neuronal replacement in the former, but neuronal addition in the latter. In contrast, our analysis indicates that PNN makes qualitatively similar contributions to both regions, but that the overall structural contribution to the $\mathrm{OB}$ is nearly two-fold larger.

Our findings demonstrate that neurogenesis during the juvenile period between 2 weeks and 2 months of age plays an essential structural developmental role by continually and robustly adding to the total adult compliment of granule neurons. This contrasts with neurogenesis in mature adults older than 2 months, in which there is no net addition of neurons and incorporation of newly generated neurons must be balanced by neuronal loss. This difference suggests that juvenile neurogenesis may subserve somewhat different functions from neurogenesis in the fully mature adult brain (e.g., Wei et al., 2011), and that studies of neurogenesis conducted on juvenile rodents may be examining developmental events. Seemingly conflicting results in the literature may be due to differences in experimental design that involve manipulations of neurogenesis during periods of pronounced juvenile increases in granule neuron number prior to 2 months of age as compared with studies conducted after 3 months of age where there is stability of total granule neuron number. Furthermore, our findings argue that the age of the animal, not just the age of the granule cells, is a critical consideration with regards to the number of proliferating cells that are likely to be affected by a particular manipulation and, ultimately, to inferences about their functional significance. This information is also important for selecting appropriately aged animals for experimental investigation, even for researchers not directly interested in PNN.

We conducted a behavioral analysis of the same transgenic loss of function mouse model used for the morphological experiments (DNMT1-cKO mice), which have a near complete ablation of all PNN. Our analysis indicated that young adult (3-5 months old) DNMT1-cKO mice exhibited a very specific set of sex-dependent cognitive changes. Standard contextual fear conditioning was intact (Figure 4B), as was fear to an auditory cue (Figure 4C) and generalization of fear to both similar and distinct contexts (Figures 7D and 8D). We did, however, observe increased fear generalization to a novel auditory stimulus (Figure 4D) as well as a sex-dependent phenotype, with an impairment in incidental contextual learning (Figure 6D), an enhancement in contextual fear discrimination learning (Figures 7F,G) and a reduced reliance on olfactory cues (Figures $\mathbf{8 F , G}$ ). We observed sex differences in control mice that were eliminated by loss of PNN, in the case of contextual discrimination learning as well sex-specific effects of the ablation, in the case of incidental contextual learning. The reduced reliance on olfactory cues exhibited both of these patterns, with the former pattern expressed in the novel context and the latter being expressed in the fear conditioned context.

The prominent sex interactions observed here are consistent with the sexually dimorphic nature of the DG, which exhibits sex differences in synaptic connectivity and plasticity as well as differences in hormone receptors and sensitivity to neurosteroids (Parducz and Garcia-Segura, 1993; Maren et al., 1994; Galea, 2008). It is also consistent with recent findings that OB PNN is important for sex-specific functions such as maternal behavior and male aggression (Sakamoto et al., 2011). Cahill (2006) pointed to the necessity of exploring sex differences in neural function "to fully understand a host of brain disorders with sex differences in their incidence and/or nature." Adolescence is a "core risk" period for the development of mental disorders, including anxiety disorders and depression, that emerge in a sexually dimorphic manner with an average age of onset that corresponds quite well to the transition from juvenile to adult neurogenesis (Hayward and Sanborn, 2002; Beesdo et al., 2009). We found that the major structural contribution of PNN occurs during sexual maturation, suggesting that juvenile neurogenesis is likely a key mediator of sex differences in brain function both in healthy individuals and in psychopathological conditions. 
Increased fear generalization to a novel auditory stimulus is consistent with a role for impaired PNN in psychopathology, as this is a hallmark of anxiety-related disorders (Sahay et al., 2007; Lissek et al., 2010). This finding may also provide evidence for a pattern separation role for PNN, as has recently been proposed (Sahay et al., 2011b). The enhancement of contextual discrimination learning, however, indicates exactly the opposite, i.e., it is suggestive of improved pattern separation ability. Enhanced contextual discrimination learning in DNMT1-cKO mice is opposite to recent findings that disruption of adult neurogenesis impairs context discrimination (Scobie et al., 2009; Tronel et al., 2010) and increasing adult neurogenesis improves it (Sahay et al., 2011a). Our near complete ablation of PNN, together with the greater difficulty of the discrimination and the absence of odor as a discriminative stimulus (see below) may have contributed to the opposite findings in the present study. Furthermore, our findings suggest that potential involvement of PNN in pattern separation may differ depending on whether the pattern to be separated is a discrete stimulus or a more complex multimodal stimulus. This would be consistent with the different roles that the hippocampus plays in cue and contextual conditioning (e.g., Kim and Fanselow, 1992).

Ablation of adult neurogenesis has been previously shown to enhance working memory in a radial arm maze task when repetitive information was presented in a single day (Saxe et al., 2007). This was interpreted as evidence for a temporal integration function, whereby events experienced close in time are more strongly bound together due to the enhanced plasticity of a cohort of immature granule cells (Aimone et al., 2006; Deng et al., 2010). This process interferes with distinguishing between events experienced close in time and release from this interference after ablation of adult neurogenesis allows for enhanced discrimination. The present findings of enhanced contextual discrimination learning after ablation of PNN may, therefore, provide further evidence for a temporal integration function, as both contexts were highly similar and were presented on each day of discrimination training.

The specific impairment of incidental contextual learning indicates that PNN may be particularly important for contextual learning in the absence of the amygdala-dependent

\section{REFERENCES}

Aimone, J. B., Deng, W., and Gage, F. H. (2011). Resolving new memories: a critical look at the dentate gyrus, adult neurogenesis, and pattern separation. Neuron 70, 589-596.

Aimone, J. B., Wiles, J., and Gage, F. H. (2006). Potential role for adult neurogenesis in the encoding of time in new memories. Nat. Neurosci. 9, 723-727.

Akirav, I., and Richter-Levin, G. (1999). Priming stimulation in the basolateral amygdala modulates synaptic plasticity in the rat dentate gyrus. Neurosci. Lett. 270, 83-86.

neuromodulatory effects foot-shock (Akirav and Richter-Levin, 1999; McGaugh, 2004; Huff et al., 2006). This could be mediated by the enhanced capacity for synaptic plasticity of the immature postnatally generated granule cells (Snyder et al., 2001; Saxe et al., 2006; Ge et al., 2007), and/or their potential preferential recruitment into learning and memory circuits (Ramirez-Amaya et al., 2006; Kee et al., 2007), although see (Stone et al., 2010). Additionally, this finding may provide further evidence for a temporal integration function of PNN. In this case, temporal integration may be necessary to bind together a brief, weakly encoded exploratory experience into a stable contextual representation.

The reduced reliance on olfactory cues we observed after ablation of PNN is consistent with previous findings of impaired olfactory-based fear conditioning after adult neurogenesis ablation when odor was used as an explicit cue (Valley et al., 2009). In the present experiment odor was only one of several sensory modalities that defined the training and novel contexts. The normal level of contextual fear and equivalent generalization prior to the odor switch indicates that PNN ablation may have increased reliance on auditory, visual, and tactile cues at the expense of olfactory cues. These findings also provide cautionary evidence against purely hippocampus-based interpretations of transgenic methodologies that affect both OB and DG PNN, particularly in contextual discrimination tasks that use different odors to define the contexts (Scobie et al., 2009; Tronel et al., 2010; Sahay et al., 2011a). In the present study, we attempted to avoid this confound as much as possible by keeping olfactory cues constant for the hippocampus-based tasks.

Recent computational work has argued that as postnatally generated granule cells mature they come to support high information encoding of the stimulus dimensions that were encountered during their immature stage (Aimone et al., 2011). Viewed in the context of the critical structural role of juvenile neurogenesis, such a process takes on new meaning: it provides a mechanism for early postnatal experience to optimize information encoding in the adult brain. This basic concept was proposed in the earliest reports of PNN (Altman and Das, 1965) and indicates that future research focused on juvenile neurogenesis may add important new insights into the functional significance of PNN as a whole.

Cameron, H. A., and Mckay, R. D. (2001). Adult neurogenesis produces a large pool of new granule cells in the dentate gyrus. J. Comp. Neurol. 435, 406-417.

Cowan, W. M., Fawcett, J. W., O’Leary, D. D., and Stanfield, B. B. (1984). Regressive events in neurogenesis. Science 225, 1258-1265.

Deng, W., Aimone, J. B., and Gage, F. H. (2010). New neurons and new memories: how does adult hippocampal neurogenesis affect learning and memory? Nat. Rev. Neurosci. 11, 339-350.

Drew, M. R., Denny, C. A., and Hen, R. (2010). Arrest of adult hippocampal neurogenesis in mice impairs single- but not multiple-trial contextual fear conditioning. Behav. Neurosci. 124, 446-454.

Fan, G., Beard, C., Chen, R. Z., Csankovszki, G., Sun, Y., Siniaia, M., Biniszkiewicz, D., Bates, B., Lee, P. P., Kuhn, R., Trumpp, A., Poon, C., Wilson, C. B., and Jaenisch, R. (2001). DNA hypomethylation perturbs the function and survival of CNS neurons in postnatal animals. J. Neurosci. 21, 788-797.

Fanselow, M. S. (1990). Factors governing one-trial contextual conditioning. Anim. Learn. Behav. 18, 264-270.

Cahill, L. (2006). Why sex matters for neuroscience. Nat. Rev. Neurosci. 7, 477-484.
Galea, L. A. (2008). Gonadal hormone modulation of neurogenesis in the 
dentate gyrus of adult male and female rodents. Brain Res. Rev. 57, 332-341.

Garcia, A. D., Doan, N. B., Imura, T., Bush, T. G., and Sofroniew, M. V. (2004). GFAP-expressing progenitors are the principal source of constitutive neurogenesis in adult mouse forebrain. Nat. Neurosci. 7, 1233-1241.

Ge, S., Yang, C. H., Hsu, K. S., Ming, G. L., and Song, H. (2007). A critical period for enhanced synaptic plasticity in newly generated neurons of the adult brain. Neuron 54, 559-566.

Gundersen, H. J., Bendtsen, T. F., Korbo, L., Marcussen, N., Moller, A., Nielsen, K., Nyengaard, J. R., Pakkenberg, B., Sorensen, F. B., Vesterby, A., and West, M. J. (1988). Some new, simple and efficient stereological methods and their use in pathological research and diagnosis. APMIS 96, 379-394.

Hayward, C., and Sanborn, K. (2002). Puberty and the emergence of gender differences in psychopathology. J. Adolesc. Health 30, 49-58.

Huff, N. C., Frank, M., WrightHardesty, K., Sprunger, D., Matus-Amat, P., Higgins, E., and Rudy, J. W. (2006). Amygdala regulation of immediate-early gene expression in the hippocampus induced by contextual fear conditioning. J. Neurosci. 26, 1616-1623.

Imayoshi, I., Sakamoto, M., Ohtsuka, T., Takao, K., Miyakawa, T., Yamaguchi, M., Mori, K., Ikeda, T., Itohara, S., and Kageyama, R. (2008). Roles of continuous neurogenesis in the structural and functional integrity of the adult forebrain. Nat. Neurosci. 11, 1153-1161.

Imura, T., Kornblum, H. I., and Sofroniew, M. V. (2003). The predominant neural stem cell isolated from postnatal and adult forebrain but not early embryonic forebrain expresses GFAP. J. Neurosci. 23, 2824-2832.

Jackson-Grusby, L., Beard, C., Possemato, R., Tudor, M., Fambrough, D., Csankovszki, G., Dausman, J., Lee, P., Wilson, C., Lander, E., and Jaenisch, R. (2001). Loss of genomic methylation causes p53dependent apoptosis and epigenetic deregulation. Nat. Genet. 27, 31-39.

Jacobs, N. S., Cushman, J. D., and Fanselow, M. S. (2010). The accurate measurement of fear memory in Pavlovian conditioning: resolving the baseline issue. J. Neurosci. Methods 190, 235-239.

Jaenisch, R. (1997). DNA methylation and imprinting: why bother? Trends Genet. 13, 323-329.

Kee, N., Teixeira, C. M., Wang, A. H., and Frankland, P. W. (2007). Preferential incorporation of adult-generated granule cells into spatial memory networks in the dentate gyrus. Nat. Neurosci. 10, 355-362.

Kempermann, G., Gast, D., Kronenberg, G., Yamaguchi, M., and Gage, F. H. (2003). Early determination and long-term persistence of adult-generated new neurons in the hippocampus of mice. Development 130, 391-399.

Kim, J. J., and Fanselow, M. S. (1992). Modality-specific retrograde amnesia of fear. Science 256, 675-677.

Lagace, D. C., Whitman, M. C., Noonan, M. A., Ables, J. L., Decarolis, N. A., Arguello, A. A., Donovan, M. H., Fischer, S. J., Farnbauch, L. A., Beech, R. D., Dileone, R. J., Greer, C. A., Mandyam, C. D., and Eisch, A. J. (2007). Dynamic contribution of nestin-expressing stem cells to adult neurogenesis. J. Neurosci. 27, 12623-12629.

Lazarini, F., and Lledo, P. M. (2011). Is adult neurogenesis essential for olfaction? Trends Neurosci. 34, 20-30.

Lissek, S., Rabin, S., Heller, R. E., Lukenbaugh, D., Geraci, M., Pine, D. S., and Grillon, C. (2010) Overgeneralization of conditioned fear as a pathogenic marker of panic disorder. Am. J. Psychiatry 167, 47-55.

Maren, S., De Oca, B., and Fanselow, M. S. (1994). Sex differences in hippocampal long-term potentiation (LTP) and Pavlovian fear conditioning in rats: positive correlation between LTP and contextual learning. Brain Res. 661, 25-34.

McGaugh, J. L. (2004). The amygdala modulates the consolidation of memories of emotionally arousing experiences. Annu. Rev. Neurosci. 27, 1-28.

McHugh, T. J., Jones, M. W., Quinn, J. J., Balthasar, N., Coppari, R., Elmquist, J. K., Lowell, B. B. Fanselow, M. S., Wilson, M. A., and Tonegawa, S. (2007). Dentate gyrus NMDA receptors mediate rapid pattern separation in the hippocampal network. Science 317, 94-99.

Ninkovic, J., Mori, T., and Gotz, M. (2007). Distinct modes of neuron addition in adult mouse neurogenesis. J. Neurosci. 27, 10906-10911.

Novak, A., Guo, C., Yang, W., Nagy, A., and Lobe, C. G. (2000). Z/EG, a double reporter mouse line that expresses enhanced green fluorescent protein upon Cre-mediated excision. Genesis 28, 147-155.

Parducz, A., and Garcia-Segura, L. M. (1993). Sexual differences in the synaptic connectivity in the rat dentate gyrus. Neurosci. Lett. 161, 53-56.

Petreanu, L., and Alvarez-Buylla, A. (2002). Maturation and death of adult-born olfactory bulb granule neurons: role of olfaction. J. Neurosci. 22, 6106-6113.

Quinn, J. J., Wied, H. M., Liu, D. and Fanselow, M. S. (2009). Posttraining excitotoxic lesions of the dorsal hippocampus attenuate generalization in auditory delay fear conditioning. Eur. J. Neurosci. 29, 1692-1700.

Ramirez-Amaya, V., Marrone, D. F., Gage, F. H., Worley, P. F., and Barnes, C. A. (2006). Integration of new neurons into functional neural networks. J. Neurosci. 26, 12237-12241.

Rogers, D. C., Fisher, E. M., Brown, S. D., Peters, J., Hunter, A. J., and Martin, J. E. (1997). Behavioral and functional analysis of mouse phenotype: SHIRPA, a proposed protocol for comprehensive phenotype assessment. Mamm. Genome 8, 711-713.

Rudy, J. W., Huff, N. C., and MatusAmat, P. (2004). Understanding contextual fear conditioning: insights from a two-process model. Neurosci. Biobehav. Rev. 28, 675-685.

Sahay, A., Drew, M. R., and Hen, R. (2007). Dentate gyrus neurogenesis and depression. Prog. Brain Res. 163 , 697-722.

Sahay, A., Scobie, K. N., Hill, A. S., O'Carroll, C. M., Kheirbek, M. A., Burghardt, N. S., Fenton, A. A., Dranovsky, A., and Hen, R. (2011a). Increasing adult hippocampal neurogenesis is sufficient to improve pattern separation. Nature 472, 466-470.

Sahay, A., Wilson, D. A., and Hen, R. (2011b). Pattern separation: a common function for new neurons in hippocampus and olfactory bulb. Neuron 70, 582-588.

Sakamoto, M., Imayoshi, I., Ohtsuka, T., Yamaguchi, M., Mori, K., and Kageyama, R. (2011). Continuous neurogenesis in the adult forebrain is required for innate olfactory responses. Proc. Natl. Acad. Sci. U.S.A. 108, 8479-8484.
Saxe, M. D., Battaglia, F., Wang, J. W., Malleret, G., David, D. J., Monckton, J. E., Garcia, A. D., Sofroniew, M. V., Kandel, E. R., Santarelli, L., Hen, R., and Drew, M. R. (2006). Ablation of hippocampal neurogenesis impairs contextual fear conditioning and synaptic plasticity in the dentate gyrus. Proc. Natl. Acad. Sci. U.S.A. 103, 17501-17506.

Saxe, M. D., Malleret, G., Vronskaya, S., Mendez, I., Garcia, A. D., Sofroniew, M. V., Kandel, E. R., and Hen, R. (2007). Paradoxical influence of hippocampal neurogenesis on working memory. Proc. Natl. Acad. Sci. U.S.A. 104, 4642-4646.

Scobie, K. N., Hall, B. J., Wilke, S. A., Klemenhagen, K. C., FujiiKuriyama, Y., Ghosh, A., Hen, R. and Sahay, A. (2009). Kruppellike factor 9 is necessary for late-phase neuronal maturation in the developing dentate gyrus and during adult hippocampal neurogenesis. J. Neurosci. 29, 9875-9887.

Shors, T. J., Townsend, D. A., Zhao, M., Kozorovitskiy, Y., and Gould, E. (2002). Neurogenesis may relate to some but not all types of hippocampal-dependent learning. Hippocampus 12, 578-584.

Snyder, J. S., Kee, N., and Wojtowicz, J. M. (2001). Effects of adult neurogenesis on synaptic plasticity in the rat dentate gyrus. J. Neurophysiol. 85, 2423-2431.

Sofroniew, M. V. (2009). Molecular dissection of reactive astrogliosis and glial scar formation. Trends Neurosci. 32, 638-647.

Stone, S. S., Teixeira, C. M., Zaslavsky, K., Wheeler, A. L., MartinezCanabal, A., Wang, A. H., Sakaguchi, M., Lozano, A. M., and Frankland, P. W. (2010). Functional convergence of developmentally and adult-generated granule cells in dentate gyrus circuits supporting hippocampus-dependent memory. Hippocampus 21, 1348-1362.

Stote, D. L., and Fanselow, M. S. (2004). NMDA receptor modulation of incidental learning in Pavlovian context conditioning. Behav. Neurosci. 118, 253-257.

Tolman, E. C. (1948). Cognitive maps in rats and men. Psychol. Rev. 55, 189-208.

Tronel, S., Belnoue, L., Grosjean, N., Revest, J. M., Piazza, P. V., Koehl, M., and Abrous, D. N. (2010). Adult-born neurons are necessary for extended contextual discrimination. Hippocampus 22, 292-298.

Valley, M. T., Mullen, T. R., Schultz, L. C., Sagdullaev, B. T., and Firestein, 
S. (2009). Ablation of mouse adult neurogenesis alters olfactory bulb structure and olfactory fear conditioning. Front. Neurosci. 3, 51. doi: 10.3389/neuro.22.003.2009

Walsh, C. P., Chaillet, J. R., and Bestor, T. H. (1998). Transcription of IAP endogenous retroviruses is constrained by cytosine methylation. Nat. Genet. 20, 116-117.

Wei, L., Meaney, M. J., Duman, R. S., and Kaffman, A. (2011). Affiliative behavior requires juvenile, but not adult neurogenesis. J. Neurosci. 31, 14335-14345.

Wiltgen, B. J., Sanders, M. J., Anagnostaras, S. G., Sage, J. R., and Fanselow, M. S. (2006). Context fear learning in the absence of the hippocampus. J. Neurosci. 26, 5484-5491.

Conflict of Interest Statement: The authors declare that the research was conducted in the absence of any commercial or financial relationships that could be construed as a potential conflict of interest.

Received: 03 December 2011; accepted: 21 January 2012; published online: 02 February 2012.

Citation: Cushman JD, Maldonado J, Kwon EE, Garcia AD, Fan G, Imura T, Sofroniew $M V$ and Fanselow MS (2012) Juvenile neurogenesis makes essential contributions to adult brain structure and plays a sex-dependent role in fear memories. Front. Behav. Neurosci. 6:3. doi: 10.3389/fnbeh. 2012.00003

Copyright (C) 2012 Cushman, Maldonado, Kwon, Garcia, Fan, Imura, Sofroniew and Fanselow. This is an open-access article distributed under the terms of the Creative Commons Attribution Non Commercial License, which permits non-commercial use, distribution, and reproduction in other forums, provided the original authors and source are credited. 